\title{
Flat photonic bands in guided modes of textured metallic microcavities
}

\author{
M. G. Salt* and W. L. Barnes \\ Thin Film Photonics Group, School of Physics, University of Exeter, Stocker Road, Exeter, Devon EX4 4QL, United Kingdom
}

(Received 28 June 1999; revised manuscript received 2 November 1999)

\begin{abstract}
A detailed experimental study of how wavelength-scale periodic texture modifies the dispersion of the guided modes of $\lambda / 2$ metal-clad microcavities is presented. We first examine the case of a solid-state microcavity textured with a single, periodic corrugation. We explore how the depth of the corrugation and the waveguide thickness affect the width of the band gap produced in the dispersion of the guided modes by Bragg scattering off the periodic structure. We demonstrate that the majority of the corrugation depths studied dramatically modify the dispersion of the lowest-order cavity mode to produce a series of substantially flat bands. From measurements of how the central frequency of the band gap varies with direction of propagation of the guided modes, we determine a suitable two-dimensional texture profile for the production of a complete band gap in all directions of propagation. We then experimentally examine band gaps produced in the guided modes of such a two-dimensionally textured microcavity and demonstrate the existence of a complete band gap for all directions of propagation of the lowest-order TE-polarized mode. We compare our experimental results with those from a theoretical model and find good agreement. Implications of these results for emissive microcavity devices such as light-emitting diodes are discussed.
\end{abstract}

\section{INTRODUCTION}

The use of planar microcavity structures to control spontaneous emission from optical devices is now a wellestablished technique. ${ }^{1-5}$ The simplest geometry is that of a pair of planar mirrors separated by a distance of the order of the wavelength of light, with the emissive species situated between the two mirrors. The mirrors may be either dielectric stacks or, in some cases, metals. ${ }^{6,7}$ It has been clearly demonstrated that the boundary conditions imposed by such planar microcavity systems can modify the spatial and spectral distribution of the emitted radiation from such devices ${ }^{4}$ and also the spontaneous emission lifetime of the emitter. ${ }^{8,9}$ However, the extent to which spontaneous emission may be controlled is limited by the planar symmetry of the microcavity. In order to modify the spontaneous emission process further, the dimensionality of the system needs to be reduced, ${ }^{10,11}$ and it is this that we have sought to do in the present study.

The introduction of wavelength-scale periodic structure into a system is known to be able to provide stop bands in the propagation of electromagnetic modes, so-called photonic band gaps (PBG's). ${ }^{12}$ A common example of this is the quarter-wave dielectric stack, where the periodic alternation of refractive index leads to a stop band in the transmission of the structure. This idea can be extended to both two and three dimensions using a range of fabrication techniques. ${ }^{13-17}$ There is currently much interest in the application of two-dimensional microstructuring to a variety of optical systems, such as providing in-plane confinement of certain microcavity modes, ${ }^{18}$ complete band gaps for surface modes, ${ }^{19}$ enhanced light extraction and out-of-plane scattering of guided modes, ${ }^{20-22}$ and feedback for organic microcavity lasers. $^{23}$ In this paper, surface microstructuring ${ }^{24}$ is used to provide band gaps for the guided modes of microcavities based on metallic mirrors. Waveguide modes are recognized as being a significant source of loss in emissive microcavity devices, most notably when the emission takes place from within a material of high refractive index. ${ }^{25}$ This loss is due to the fact that power emitted into these guided modes is usually either absorbed during propagation inside the device or eventually escapes from the cavity in a range of undesirable directions, thus reducing the overall useful efficiency of the device. Providing periodic microstructuring of one or more of the interfaces of the microcavity opens up the possibility of reducing this lost power in two ways. First, by choosing a suitable periodicity one can tailor the structure to scatter the guided light out of the device in a useful direction. ${ }^{20}$ Second, one can use the periodicity to provide a band gap in the dispersion of the guided modes at the frequency of emission, thus preventing emission into the mode initially. ${ }^{26}$ Both approaches require a detailed understanding of how the periodic microstructure may be used to modify the waveguide modes of the optical microcavity.

In contrast to our previous work on corrugated multimode cavities, ${ }^{27}$ our use here of the lowest-order cavity mode results in the corrugation producing a dramatic change to the dispersion. The band gaps produced by the surface corrugation are such that the dispersion curve now consists of a series of flat bands. Such are the widths of the band gaps generated that a full gap in all propagation directions is produced by a doubly corrugated surface for the lowest-order TE waveguide mode.

The sample design makes use of Shipley S1805 photoresist as the waveguide medium, with optically thin silver as the mirrors. Using photoresist as the waveguide medium has two main advantages: it is easily deposited by spin coating, and it can be corrugated on its upper surface by exposure to an interference pattern and subsequent development. The silver mirrors are thick enough to provide a significant reflectivity and thus form a microcavity. However, they are also thin enough to ensure that the waveguide modes are not completely confined within the cavity, but have a small leaky character, i.e. they are partially guided. This allows the mi- 
crocavity modes to be studied by observing transmission through the sample. Samples fabricated in this way yield a structure similar to many practical devices, most notably the organic light-emitting diode (LED), ${ }^{28}$ and thus provide a useful system in which a range of microstructure configurations can be explored.

Section II describes the fabrication and structure of the samples. We explain how angle-dependent reflectivity data have been used to characterize the dielectric constants, layer thickness, and surface profile of these structures in Sec. III, together with the apparatus used to study transmission through the sample. In Sec. IV, data are presented on the transmitted intensity over a range of incident optical frequencies $(\omega)$ and in-plane wavevectors $\left(k_{x}\right)$, from which the form of the dispersion curve of the guided modes is obtained. Plots of transmitted intensity as a function of $\omega$ and $k_{x}$ for the planar region of each sample are compared to those of the corrugated regions, demonstrating the clear modification to the waveguide mode dispersion that the corrugations introduce. Furthermore, we compare the width of the band gap generated by four structures of different corrugation depth and waveguide thickness. For each of the four structures, the width and position in energy of the band gap for propagation perpendicular to the corrugation grooves are compared with the width and position in energy of the band gap for propagation at $45^{\circ}$ to the corrugation grooves. From these comparisons we suggest a suitable surface profile for the provision of a complete band gap. Finally, a sample with such a suitable two-dimensional profile is fabricated and studied and is shown to provide a complete band gap for the $\mathrm{TE}_{0}$ waveguide mode.

The samples examined in this study are metal-bound dielectric waveguides. The background physics for the dispersion of waveguide modes in planar systems has been covered in our previous work. ${ }^{27}$ The introduction of Bragg scattering into the system, via some form of in-plane periodic modulation, results in the presence of counterpropagating modes. In the case of periodically textured surfaces, the presence of these counterpropagating modes does not necessarily mean that a band gap will be present in the dispersion of the waveguide modes. Figure 1 shows two possibilities that may arise for periodic modulation in only one direction, i.e., a corrugation with a peak to peak distance $\lambda_{g}$. The Bragg vector for this corrugation is denoted $k_{g}\left(=2 \pi / \lambda_{g}\right)$ and is parallel to the $k_{x}$ axis. The Brillouin-zone boundary is then at $k_{g} / 2$. Applying this corrugation to both surfaces of a waveguide, with no phase difference between the periodic interfaces, gives the cross section shown schematically in Fig. 1(a), the symmetric waveguide microcavity. For counterpropagating waveguide modes that cross at ${ }_{-}^{+} k_{g} / 2$, we can expect standing waves to be generated. The standing waves consist of two eigensolutions. Relative to the upper corrugation, the antinodes of the electric field of one solution are positioned under the peaks of the corrugation, whereas for the second solution they are positioned under the troughs. However, the symmetry of this waveguide means that the local environment for the antinodes of these two field configurations is identical, the same being true for the nodes of each standing wave. This means that the energy associated with each standing-wave eigensolution is identical, and thus no band gap exists (dotted-line crossing at ${ }_{-}^{+} k_{g} / 2$ in Fig. 1). For the
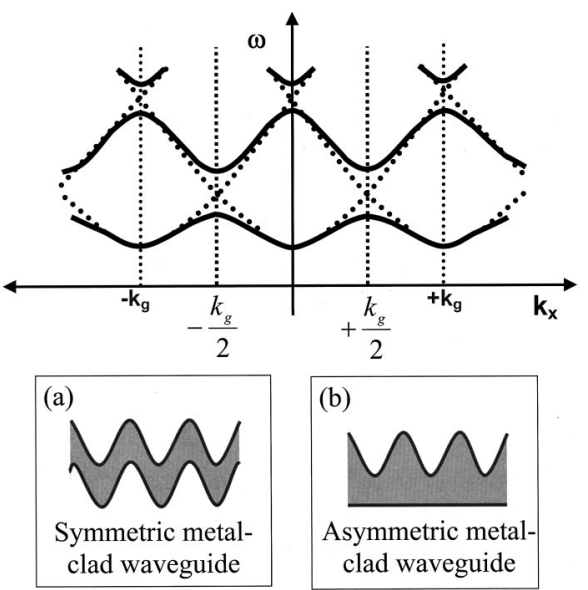

FIG. 1. An extended zone schematic of the dispersion of a waveguide mode in the singly corrugated microcavity. The Brillouin-zone boundary is indicated by the vertical dashed lines at $\pm k_{g} / 2$. For the symmetric case of inset (a), there is no energy difference between the eigensolutions at the crossing points and hence no band gap, as indicated by the heavy dotted lines. Asymmetry of the structure, as in inset (b), causes an energy difference between the two solutions to arise, leading to a band gap, as shown by the heavy black lines. Band gaps arising from second-order scattering (in the asymmetric case) are also shown. The gray regions of each inset are the cavity dielectric; the heavy dark lines are the metallic mirrors.

asymmetric case [inset Fig. 1(b)], however, the nontextured lower interface now means that there is a difference in energy between the two eigensolutions, so that a band gap now opens in the dispersion of the waveguide mode, as indicated by the dark, continuous lines at ${ }_{-}^{+} k_{g} / 2$ in Fig. 1 . With this in mind, the samples fabricated for this study both consist of one textured and one flat mirror, thus ensuring a band gap will be formed.

\section{SAMPLE GEOMETRY AND FABRICATION}

The two samples used in this study share a common fabrication process. The waveguides consist of a layer of Shipley S1805 photoresist sandwiched between two optically thin silver mirrors, supported by a silica substrate flat to $\lambda / 10$. The mirrors are thick enough to provide good field confinement for waveguide modes while being thin enough to enable sufficient access to these modes through the top mirror and so allow the transmission of the partially guided light through the lower mirror.

Sample fabrication commenced with the evaporation of the lower silver mirror (approximately $40 \mathrm{~nm}$ thick) onto the bare silica substrate. An identical control sample was included in the evaporation process, so that optical studies of this sample could be undertaken to allow the thickness of the silver layers to be determined. The sample was then spincoated with photoresist. In order to provide a $\lambda / 2$ cavity, it was found necessary to reduce the concentration of the resist. To this end, $4 \mathrm{ml}$ of resist was mixed with $5 \mathrm{ml}$ of Microposit EC solvent, this solution then being spun at $1500 \mathrm{rpm}$. The sample was then baked at $95^{\circ} \mathrm{C}$ for $30 \mathrm{~min}$ to drive off the majority of the remaining solvent from the resist layer.

Surface texturing of the waveguide was carried out by 
exposing the resist layer to an interference pattern, using a $\mathrm{He}-\mathrm{Cd}$ laser operating at a wavelength of $325 \mathrm{~nm}$ as the light source. ${ }^{29}$ Resist solubility in the developer solution depends on the total energy of the exposure. With this particular resist, the higher the exposure, the more readily the resist will dissolve. The result for a single exposure, upon immersion in the Microposit developer, is a single corrugation. The surface thus produced is a diffraction grating. The peak-to-peak distance of the grating ( $\gamma_{g}$, the grating pitch) can be controlled by changing the angle of incidence of the two interfering beams with respect to the normal of the resist layer. It is this grating pitch that determines the position of the Brillouin-zone boundary in the dispersion of the waveguide modes and hence the in-plane wave vector at which the band gap occurs. Broadly speaking, the depth of the corrugation is controlled by the total energy of exposure. Hence, longer exposure times and higher laser powers generally give deeper corrugations. More complex, "two-dimensional" patterns can be made by turning the substrate through some angle, about the normal to the plane of the resist layer, after the first exposure, and then reexposing. The result is then a convolution of the two exposures. ${ }^{30}$

The treatment of the resist by thermal baking and deep uv exposure after development of the required surface profile was found to be unnecessary, with the resist surface being robust and stable enough for all subsequent processes and study without further treatment. This was very useful, as experience has shown that the optical absorption of the resist increases significantly during heating, which in the present study would prove detrimental to both the waveguiding properties of the microcavity and to the intensity of the transmitted light. Finally, after surface development, an upper silver layer of approximately $40 \mathrm{~nm}$ thickness was evaporated to complete the waveguide structure. A bare silica control sample was also included with this evaporation.

The first sample studied consisted of five regions. One region was left unexposed to act as a comparison between corrugated and uncorrugated regions. The other four regions were textured with a single corrugation of the same pitch but different depths. A schematic of the cross section of such a corrugated waveguide is shown in Fig. 1(b). The difference depths were achieved by "stepping" the exposure. A mask was used to ensure that approximately one-quarter of the total area to be exposed was uncovered. A 60-s exposure was then made on this region. The mask was then moved so as to reveal the next quarter of the area, leaving the first quarter also uncovered. A second 60-s exposure was then made over both these regions. This method was continued until all four regions have been exposed, with exposures of 60, 120, 180, and $240 \mathrm{~s}$, respectively. We denote these regions 1 to 4 , from least to greatest exposure. A secondary effect of the depth variation thus produced was that the overall effective thickness of the waveguide in each region was different, with the planar region being thickest and the region of greatest exposure being thinnest. This aspect is discussed further in the experimental results section.

The second sample consisted of a planar region and a region of double exposure. A small section of the sample surface was masked, so as to remain planar. The sample was then exposed for $165 \mathrm{~s}$. After this first exposure the sample was rotated through $90^{\circ}$ about the normal to the plane of the

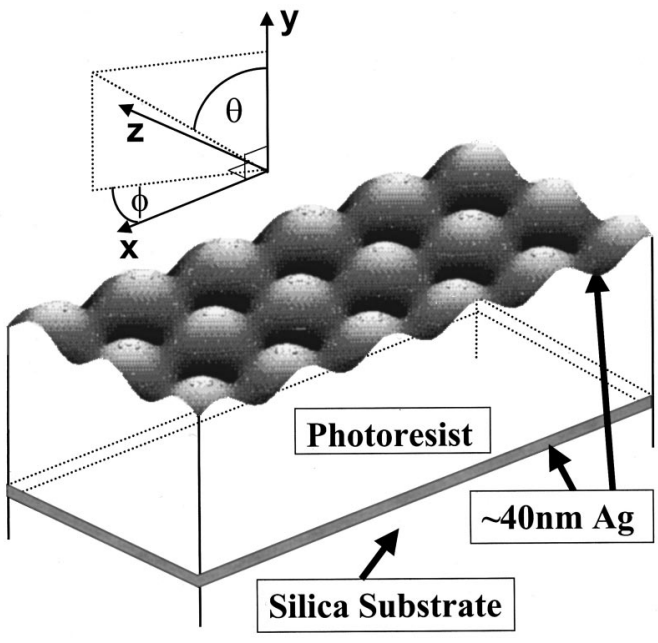

FIG. 2. A representation of the textured waveguide of the second sample studied. Two exposures made at $90^{\circ}$ to each other lead to a surface profile that provides periodicity in two orthogonal directions and to the possibility of a complete waveguide mode band gap.

substrate and a second exposure was made, again for $165 \mathrm{~s}$. The result, when developed, was a two-dimensional pattern, or "bigrating." Again, the thickness of the patterned region is less than that of the planar region. A schematic of this sample geometry, together with the coordinate system used in this paper, is shown in Fig. 2. In the single corrugation case of the first sample, the $x$ direction is perpendicular to the grating grooves.

\section{CHARACTERIZING THE SAMPLES}

The two samples were silver-coated simultaneously for both the upper and lower silver mirrors. This meant that characterization of the two control samples included in the evaporation process, one for the lower mirror and one for the upper, gave information on the thickness of the two silver layers for both the singly periodic and doubly periodic samples. Measurements of TM-polarized absolute reflectivity versus incident angle for both control samples were then taken for TM-polarized laser light having a wavelength of $612 \mathrm{~nm}$. A theoretical model of the reflectivity, based on Fresnel's formulas, ${ }^{31}$ was fitted to these data, yielding values for the silver thickness. Both silver layers were found to have a thickness of $38 \pm 3 \mathrm{~nm}$.

Information on the surface profiles of the corrugated regions was obtained by making absolute reflectivity measurements from small sections of each region that had been further coated with an optically thick (approximately $150 \mathrm{~nm}$ ) layer of silver. These areas of silver were evaporated on top of the previously optically thin top silver mirrors after the transmission data had been acquired surface plasmonpolariton (SPP) resonances hold the key to obtaining the surface profiles of the corrugations. ${ }^{32}$ The required in-plane wave-vector matching of incident TM-polarized light to the SPP mode is achieved via the addition (or substraction) of integer multiples of the Bragg wave vector $k_{g}\left(=2 \pi / \lambda_{g}\right)$ to (or from) the in-plane wave vector of the incident light. A minimum in reflectivity occurs when energy is coupled from the incident light into the SPP mode. The exact form of these 


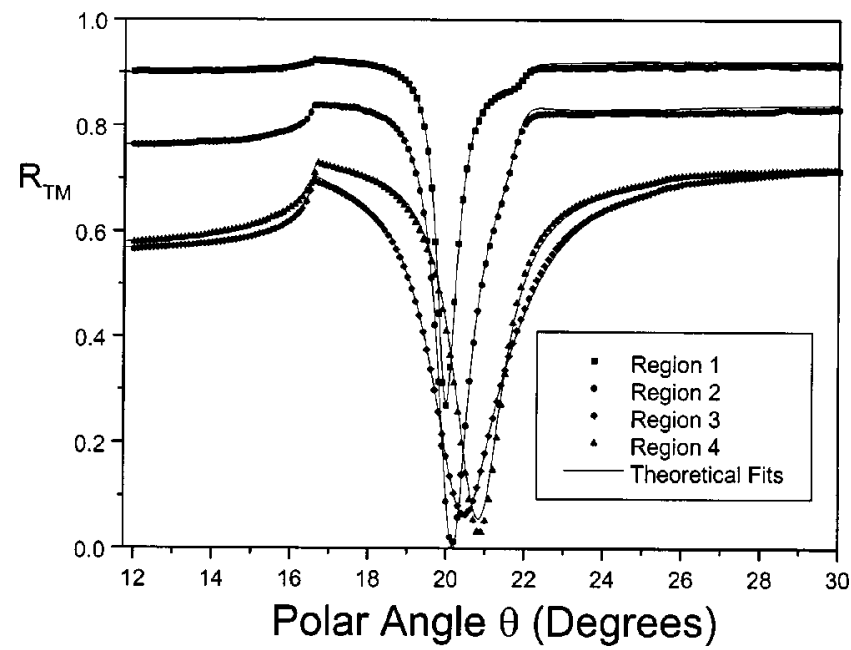

FIG. 3. TM-polarized angle-dependent reflectivity data taken for each of the four corrugated regions of the sample. The plane of incidence was aligned so as to be perpendicular to the corrugation grooves. The wavelength of the incident laser light used for each of the scans was $543.5 \mathrm{~nm}$ from a He-Ne laser. The corrugation profile parameters used to fit theory to the data were as follows. Region 1: grating pitch, $\lambda_{g}=759.8 \pm 1 \mathrm{~nm}$; fundamental amplitude, $a_{0}=13.7$ $\pm 0.5 \mathrm{~nm}$; first harmonic amplitude, $a_{1}=1.0 \pm 0.1 \mathrm{~nm}$, relative phase $\phi_{1}=90^{\circ}$. Region 2: $\lambda_{g}=759.8 \pm 1 \mathrm{~nm}, a_{0}=25.2 \pm 0.5 \mathrm{~nm}$, $a_{1}=1.25 \pm 0.7 \mathrm{~nm}$, relative phase $\phi_{1}=90^{\circ}$. Region 3: $\lambda_{g}=759.8$ $\pm 1 \mathrm{~nm}, a_{0}=37.3 \pm 0.5 \mathrm{~nm}, a_{1}=2.4 \pm 1.2 \mathrm{~nm}$, relative phase $\phi_{1}$ $=-90^{\circ}, a_{2}=1.6 \pm 0.3 \mathrm{~nm}$, relative phase $\phi_{2}=0^{\circ}$. Region 4: $\lambda_{g}$ $=760.8 \pm 1 \mathrm{~nm}, a_{0}=37.0 \pm 0.5 \mathrm{~nm}, a_{1}=2.7 \pm 1.5 \mathrm{~nm}, \quad$ relative phase $\phi_{1}=-90^{\circ}$.

resonances is very sensitive to the shape of the surface profile, and hence a study of such resonances leads to an accurate description of this profile.

Figure 3 shows the results of such measurements for the different regions of the singly corrugated sample. The region from which each data set was taken is given by the graph legend. TM-polarized light of wavelength $543.5 \mathrm{~nm}$, from a He-Ne laser, was incident upon each region of the sample. The plane of incidence was perpendicular to the grating grooves, i.e., parallel to the $x$ axis. Two distinct features are common to all four scans. The first is a rise in intensity up to a critical edge located at an incident angle of approximately $16.6^{\circ}$. This corresponds to the point where the polar angle of the first diffracted order becomes greater than $90^{\circ}$ and ceases to propagate. The corrugation pitch can be accurately calculated from this feature and was found to be $759.8 \mathrm{~nm}$ for the first three scans (regions 1-3) and $760.8 \mathrm{~nm}$ for the final scan (region 4), which was taken later than the first three. This very slight discrepancy can be explained by a small error in fixing the plane of incidence, so that it is not perpendicular to the grating grooves, and by an error in fixing the $\theta=0^{\circ}$ position. This error in the position of this feature is of the order of $0.1^{\circ}$. The second feature is a combination of two intensity minima. The first, and deepest, minimum is associated with a Bragg scattering of the incident light by $-k_{g}$, providing coupling to the air/silver SPP. The second minimum gives rise to a "distortion" to the right-hand side of the first intensity minimum. This is a coupling to the SPP via a Bragg scattering of the incident light by $-2 k_{g}$. By fitting theory to these data, the two resonances provide both the amplitudes and relative phases of the fundamental, first, and second harmonics for a Fourier representation of the surface profile of the form:

$z(x)=a_{0} \sin \left(k_{g} x\right)+a_{1} \sin \left(2 k_{g} x+\phi_{1}\right)+a_{2} \sin \left(3 k_{g} x+\phi_{2}\right)$.

The profile parameters used to fit to the data are given in the caption to Fig. 3. It should be noted, however, that these parameters describe the profile of the surface at the air/silver interface after the evaporation of a comparatively thick layer of silver. It is therefore reasonable to assume that there may be some slight differences between the shape of this surface and that of the silver/resist interface that is responsible for the creation of the band gap. Previous work ${ }^{27}$ has found that the addition of the optically thick layer of silver for the purposes of SPP surface profile characterization generally reduces the depth of such corrugations by a few nanometers. However, the effect is not dramatic and, as is shown later, good agreement between experiment and theory for transmission through such structures can be obtained by very slight reduction of the fundamental amplitudes given in the caption to Fig. 3. Surface profiles determined in this way have been shown to be consistent with (and sometimes more accurate than) those determined using surface probe techniques. $^{33}$

The theoretical model uses a scattering matrix technique ${ }^{34}$ based on a conical version ${ }^{35}$ of the differential formalism of Chandezon et al. ${ }^{36}$ This approach transforms the optical fields into a curvilinear coordinate system, in effect "flattening" the corrugation profile. This then simplifies the boundary conditions of the system, so that all the outgoing field amplitudes can be calculated using Maxwell's equations, allowing the optical characteristics to be fully modeled. Of most interest here are the fundamental amplitudes $a_{0}$, as they are the main contributors to the widths of the band gaps with this sample design.

Modeling the doubly periodic surface profile of the second sample via similar reflectivity measurements has proven to be beyond our computational resources, due to the twodimensional nature of the surface. However, measurements of the critical edge positions for the two crossed gratings yielded values for the pitch of each grating. These are $761.4 \pm 1$ and $762.1 \pm 1 \mathrm{~nm}$. The azimuthal angle $\phi$ between the two gratings was found to be $88^{\circ} \pm 0.2^{\circ}$, by measuring the position of the diffracted orders.

The apparatus and method used to study transmission through the sample have been given elsewhere. ${ }^{27}$ In brief, the sample is mounted on a rotation stage, which allows angle of incidence (polar angle $\theta$ ) and azimuthal angle $\phi$ (both defined in Fig. 2) to be set to the desired values. A monochromator, with an approximate spectral half-width of $1.5 \mathrm{~nm}$ and beam divergence of $0.04^{\circ}$, provided the required wavelength of incident light. The polarization of the incident and transmitted light was set to either TM or TE using polarizers. Transmitted and reference signals were measured using photomultiplier detectors. The scan procedure was automated, with wavelength selection and angle of incidence both controlled by computer. The final dispersion curves (gray-scale maps, Figs. 4, 7, and 8) are produced by defining the range of $\omega$ and $k_{x}$ over which one wishes to scan, including the 
number of data points (resolution) required. Scanning proceeded by fixing the in-plane wave vector at its lowest value, by adjusting the polar angle of incidence $\theta$, and by sweeping through the preset $\omega$ range. The computer then fixes the next lowest value for the in-plane wave vector and the $\omega$ range is swept again. This is repeated for each value of in-plane wave vector until a complete $\omega$-versus- $k_{x}$ map is constructed over the desired range.

\section{EXPERIMENTAL RESULTS AND DISCUSSION}

Figure 4 shows the transmitted intensity as a function of both $\omega$ and $k_{x}$ for the planar region and for three of the four different depths of single corrugation from the first sample. Data for region 4 has not been included, as it is very similar to that of region 3. The $\omega$ range spans the whole of the visible (from $\lambda=400$ to $\lambda=800 \mathrm{~nm}$ ). The darker areas of the gray scales indicate high transmission. The diagonal dotted line on each plot indicates the $\theta=65^{\circ}$ position for the angle of incidence. Beyond this angle the sample holder cuts off the incident beam, so that points plotted to the right of this line are of no relevance.

Figure 4(a) shows the transmitted intensity for the planar region of the sample. The incident and detected light were unpolarized. This plot clearly shows the expected dispersion of the $\lambda / 2$ guided mode. The polarization splitting of the mode at higher in-plane wave vectors is clearly shown, this splitting being due to the difference in reflectivity between TM and TE polarizations at the cavity interfaces. At high $\omega$ values, coupling (a darkening of the gray scale) to the $\lambda$ mode can just be seen, but is very weak owing to the resonant frequency of this mode being out of range of the scanning equipment. All transmission data have been normalized with respect to the incident intensity, the maximum transmitted intensity for this plot being $33 \%$ at $k_{x}=0 \mu \mathrm{m}^{-1}$ and $\omega$ $\approx 1.48 \mu \mathrm{m}^{-1}(\approx 1.99 \mathrm{eV})$. The error associated with this transmission measurement is difficult to quantify, as accuracy tends to scale with signal strength, but we estimated an error of approximately $\pm 4 \%$.

Figure 4(b) shows the effect the shallowest corrugation, region 1, has on the dispersion for TE-polarized light. The plane of incidence is parallel to the $x$ axis. The Brillouinzone boundary is indicated by the vertical dashed line (at $\left.k_{g} / 2\right)$. A band gap has opened at this point, due to first-order Bragg scattering. The lower band edge $\left(\omega^{-}\right)$is at $\omega$ $\approx 1.61 \mu \mathrm{m}^{-1}(\approx 2.00 \mathrm{eV})$ and is flat, with the upper band edge $\left(\omega^{+}\right)$beginning at $\omega \approx 1.75 \mu \mathrm{m}^{-1}(\approx 2.18 \mathrm{eV})$. The width of this band gap in energy is then approximately 0.14 $\mu \mathrm{m}^{-1}(0.18 \mathrm{eV})$. A secondary band gap can be seen to the upper right of the main gap. This has been generated by second-order Bragg scattering, is approximately $0.06 \mu \mathrm{m}^{-1}$ $(0.08 \mathrm{eV})$ wide, and is situated at $k_{x}=k_{g}$. The same band gap is also just visible, centered at $k_{x}=0$. It appears twice because incident light may couple to it by picking up different Bragg vectors from the corrugation (see Fig. 1). This band gap is difficult to see at this shallow depth, due to the poor coupling between the incident and transmitted light. The faint diagonal line leading from $k_{x}=0, \omega \approx 1.8 \mu \mathrm{m}^{-1}$ $(\approx 2.23 \mathrm{eV})$ to $k_{x}=k_{g} / 2, \omega \approx 2.0 \mu \mathrm{m}^{-1}(\approx 2.48 \mathrm{eV})$ and beyond is the continued dispersion of the mode due to the incident light being Bragg scattered by $+k_{g}$ into the waveguide mode.

Figure 4(c) shows the TM-polarized transmission for the same region of the sample as Fig. 4(b). The plot is more complicated due to the ability of the incident TM-polarized light to couple to SPP's propagating at the air/silver, silver/ resist, resist/silver, and silver/silica interfaces via Bragg scattering. Should these modes then share the same in-plane wave vector and frequency as the waveguide mode, distortion occurs due to coupling between the modes at these crossing points. However, upper and lower "band edges" are still discernible, as indicated by the arrows labeled $\omega^{+}$ and $\omega^{-}$for both the main $\left(k_{x}=k_{g} / 2\right)$ and secondary $\left(k_{x}\right.$ $=k_{g}$ ) band gaps. The width of these gaps is approximately $0.13 \mu \mathrm{m}^{-1}(\approx 0.16 \mathrm{eV})$ and $0.10 \mu \mathrm{m}^{-1}(\approx 0.11 \mathrm{eV})$, respectively. Thus, although band gaps have been generated in the dispersion of the TM-polarized waveguide mode, there is nevertheless coupling in the band-gap region to different SPP modes. The surfaces supporting these SPP modes have been identified by making use of the theoretical plot of Fig. 5. This plot has been generated by calculating the power dissipated by a dipole source located in the photoresist of an equivalent planar microcavity, ${ }^{37}$ using the parameters obtained from previous work ${ }^{27}$ and from the theoretically generated plots of Fig. 6 (see below). The dark regions correspond to power dissipation and occur when the modes of the microcavity are well coupled to the dipole source. Thus the gray-scale plot indicates the dispersion of the microcavity modes. The most useful aspect of this type of representation in the present context is that the plot shows all the modes supported by the microcavity over a certain range of $\omega$ and $k_{x}$, including, most importantly in this case, evanescent modes. Furthermore, by taking account of Bragg scattering by integer multiples of $k_{g}$, one can identify the Braggscattered SPP modes seen in the TM-polarized data of Fig. 4. The modes crossing the plot of Fig. 4(c) are identified as being [see Fig. 4(c)] (1) SPP silver/silica, coupled via a Bragg scatter of $-2 k_{g}$, (2) SPP silver/air, coupled via a Bragg scatter of $+k_{g}$, (3) SPP silica/silver, coupled via a Bragg scatter of $+2 k_{g}$, (4) SPP silver/air, coupled via a Bragg scatter of $+k_{g}$, (5) SPP silver/air, coupled via a Bragg scatter of $-2 k_{g}$, (6) SPP silica/silver, coupled via a Bragg scatter of $+k_{g}$, (7) $\mathrm{TM}_{0}$ waveguide mode, coupled via a Bragg scatter of $+k_{g}$, and (8) SPP silica/silver coupled via a Bragg scatter of $-3 k_{g}$. It is also possible to see that the position of the lower band edge is lower that that of the TE-polarized data, even at $k_{x}=0$. This is due to the different way the TE and TM polarizations interact with the corrugated surface topography, leading to differences in the transmission and reflection coefficients for the corrugated interfaces for each polarization.

Figures 4(d) and 4(e) show the TE- and TM-polarized transmission for the next-deepest corrugation, region 2 . The entire waveguide mode dispersion is higher in energy, indicating an overall reduction in the effective thickness of the cavity. For the TE-polarized case, the width of the main band gap at $k_{g} / 2$ has increased to $0.25 \mu \mathrm{m}^{-1}(\approx 0.31 \mathrm{eV})$. The width of the secondary gap at $k_{g}$ has also increased, to 0.19 $\mu \mathrm{m}^{-1}(\approx 0.23 \mathrm{eV})$. As coupling between the scattered modes becomes stronger, the formation of other band edges can be more clearly seen at $k_{x}=0, \omega \approx 2.10 \mu \mathrm{m}^{-1}(2.60 \mathrm{eV})$ and at 

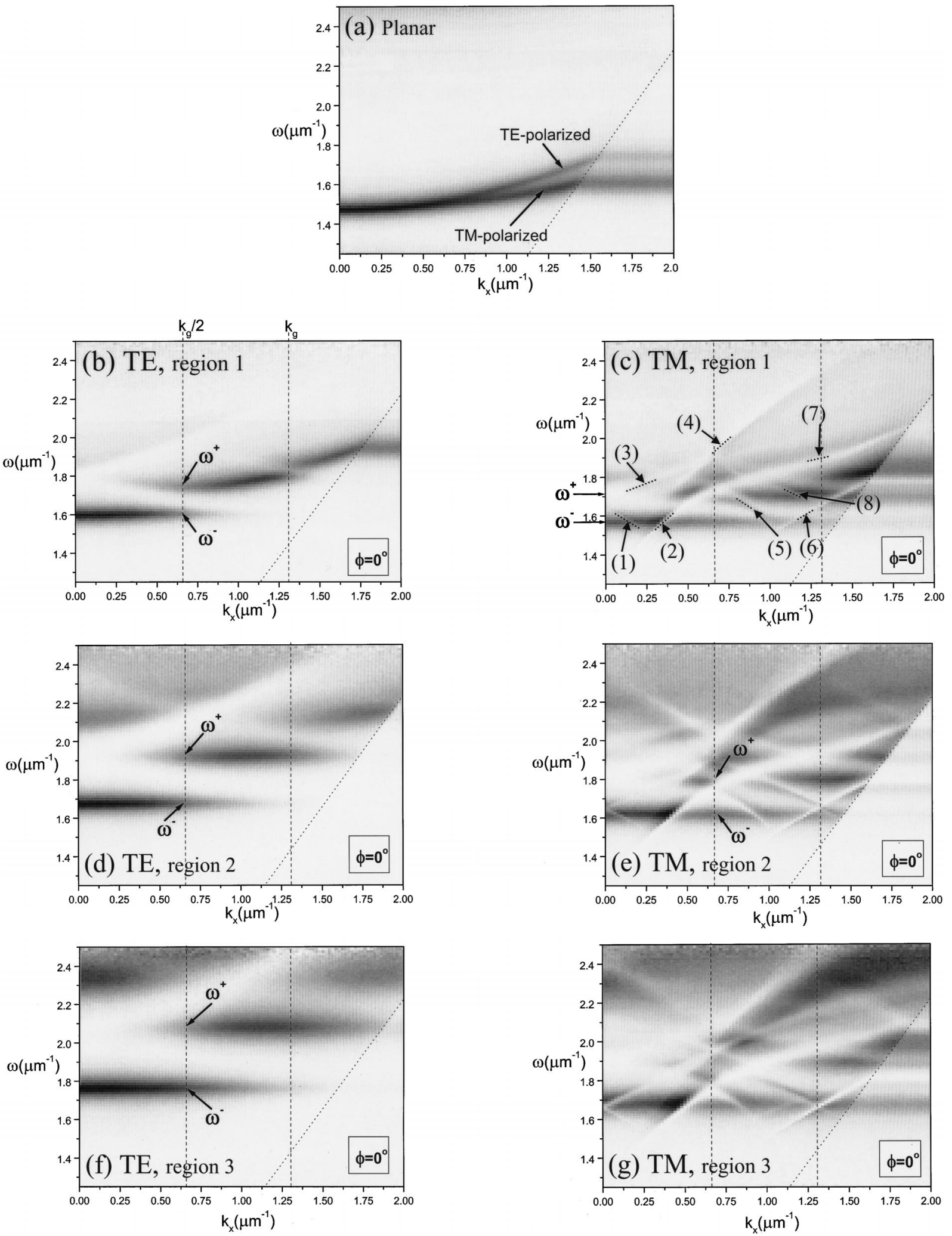

FIG. 4. Transmitted intensity as a function of frequency $\omega$ and in-plane wave vector $k_{x}$ for the planar and three of the four corrugated regions of the sample. Dark regions are high transmitted intensity, and light regions are low intensity. The diagonal dotted line on each plot indicates the polar angle $\theta=65^{\circ}$. To the right of this point the sample mount has cut off the incident beam and the data taken are of no relevance. (a) shows the dispersion of the planar region of the sample, with the TE and TM modes splitting due to the difference in reflectivity at the interfaces. (b) $-(\mathrm{g})$ show the evolution of the band gap as the corrugation depth is increased and the effective photoresist thickness is decreased. The plots are in ascending order of region number, as defined in the text. The vertical dashed lines [labeled in (b)] indicate the position of the Brillouin-zone boundary $\left(k_{g} / 2\right)$ and the position where $k_{x}=k_{g}$. As can be seen, deeper corrugations lead to larger band gaps and significantly flatter bands, although the presence of surface plasmon-polariton modes in the TM case prevents clear band edges and gaps from being seen. The effective cavity thickness also reduces with increasing region number, leading to an overall rise in the position of the waveguide modes. 


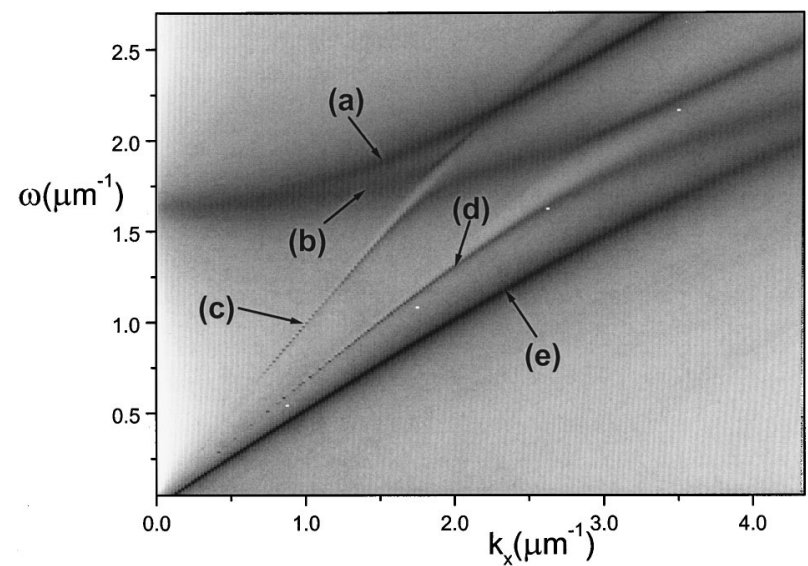

FIG. 5. This plot shows the calculated power dissipated by a dipole source located in a planar microcavity structure, based upon the parameters obtained for region 1. Darker areas are regions of higher power dissipation, the scale being logarithmic. Coupling to both guided and surface modes of the microcavity by the dipole source results in an increase in power dissipation at the value of $\omega$ and $k_{x}$ where that mode exists. The dark lines therefore trace out the dispersion of these modes. These modes are (a) $\mathrm{TE}_{0}$ waveguide mode, (b) $\mathrm{TM}_{0}$ waveguide mode, (c) SPP air/silver, (d) SPP silver/ silica, and (e) SPP photoresist/silver.

the Brillouin-zone boundary, where $\omega$ $\approx 2.24 \mu \mathrm{m}^{-1}(2.78 \mathrm{eV})$. For the TM-polarized case there is also an increase in the width of the waveguide mode band gaps to $0.18 \mu \mathrm{m}^{-1}(0.22 \mathrm{eV})$ for the main gap and 0.11 $\mu \mathrm{m}^{-1}(0.15 \mathrm{eV})$ for the secondary gap. The nature of the dispersion is further complicated by the accompanying increase in coupling to the SPP modes.

The trend of a reduction in the effective cavity thickness and an increase in the width of the band gaps continues for the final two corrugation depths, regions 3 and 4. For region 3, Fig. 4(f), the width of the TE-polarized main band gap is approximately $0.40 \mathrm{eV}$, with the width of the secondary gap at $k_{x}=k_{g}$ being about $0.32 \mathrm{eV}$. The TM-polarized mode structure, Fig. 4(g), now has a large number of well-coupled modes. It is now impossible to the band edges in the waveguide modes of such a mode structure. The TE-polarized transmission of region 4 (not shown) exhibited a form similar to that of region 3. A main band gap of approximately $0.45 \mathrm{eV}$ was observed, with the upper edge of the secondary band gap at $k_{x}=k_{g}$ beyond the $\omega$ range of the scanning equipment. The TM-polarized transmission was dominated by coupling to SPP modes in this case also.

Perhaps of greatest significance from these data is the flatness of the band structure. This makes a large range of $k_{x}$ values available for a relatively small range of frequency, i.e., greatly increasing the density of states. This has significant impact on spontaneous emission from within such a structure, as will be discussed further later in this section and also in Sec. V.

By taking the parameters from the fits to the angledependent reflectivity data of Fig. 3, it has been possible to generate theoretical plots that closely resemble those of Fig. 4. The modeling technique used to generate these plots is the same as that used to model the SPP resonances of Fig. 3. Figures 6(a) and 6(b) are typical examples of two such theoretical plots, obtained using the parameters for region 2 .

The slight differences between the experimental data and the theoretical plots are due to the constraints of the modeling technique used. It has not been possible to accurately model the presence of the 1.5 -mm-thick silica substrate used in these structures due to numerical instabilities in the code when dealing with such a comparatively thick layer. The structure used in the modeling has had to be simplified to a symmetric layer structure consisting of air/silver/photoresist/ silver/air. The dielectric constants of the photoresist layer have also been kept constant with wavelength, whereas it is known that both the real and imaginary parts increase with frequency. Due to this simplification, there are slight differences in the position, widths, and coupling strengths of the modes, but not to such an extent as to render such comparisons invalid, as the reflectivity at the interfaces is dominated by the silver layers. The absolute transmission due to the TE

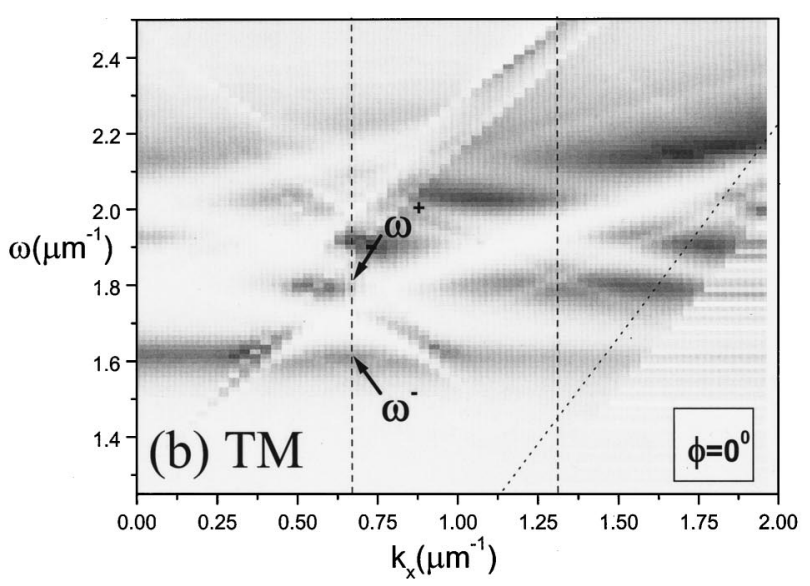

FIG. 6. Theoretically generated plots based upon the structure of region 2 of the first sample. Due to modeling constraints, the structure represented here does not include the silica substrate of the real sample. The parameters used to generate the two plots were $a_{0}=28.0$ $\pm 0.5 \mathrm{~nm}, a_{1}=1.25 \pm 0.5 \mathrm{~nm}, \phi_{1}=90^{\circ}$, average photoresist thickness $d_{\mathrm{PR}}=120 \pm 1 \mathrm{~nm}$, Drude model scattering time for silver $\tau=7.05$ $\times 10^{-15} \mathrm{~s}$, plasma frequency for silver $\omega_{p}=1.2 \times 10^{16} \mathrm{~Hz}$, silver layer thickness $d_{\mathrm{Ag}}=38 \pm 3 \mathrm{~nm}$ for both layers, and dielectric constant for photoresist $\varepsilon_{\mathrm{PR}}=2.65 \pm 0.05+0.0016 \pm 0.0004 i$. 

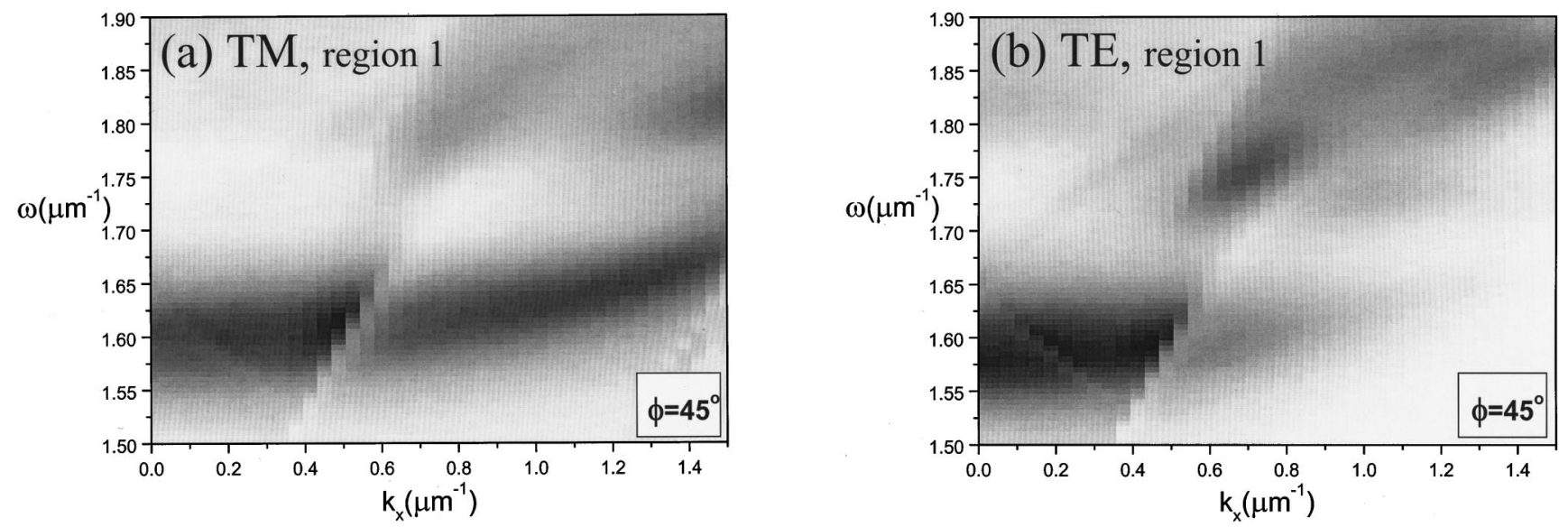

FIG. 7. Transmitted intensity as a function of frequency $\omega$ and in-plane wave vector $k_{x}$ for region 1 , with the sample rotated so that the plane of incidence is at $45^{\circ}$ to the corrugation grooves. The plane of polarization of the input and output polarizers is not rotated with the sample, remaining instead at the same orientation as in the $\phi=0^{\circ}$ study. (a) shows the TE-polarized transmission for TE-polarized incident light. The surface profile allows coupling to SPP's, which can be seen crossing the plot. (b) shows the TM-polarized transmission for TM-polarized light incident on the same region of the sample as in (a). Both plots demonstrate that even this relatively small corrugation is able to provide a definable upper and lower branch to the waveguide mode band structure at this azimuthal angle, with very little change in position for TE polarization compared with the $\phi=0^{\circ}$ case [Fig. 4(b)]

waveguide mode is highest at $k_{x}=0$, with a value of $24 \%$. For the TM guided mode this value is $11 \%$ at $k_{x}=0$. These compare well with the experimental values for region 1 of $(20 \pm 4) \%$ for the TE and $(11 \pm 4) \%$ for the TM guided mode of the sample. It should also be noted that we have found that the inaccuracies in the description of the structure do not affect the widths of the band gaps or the value of in-plane wave vector at which they occur, as these are largely determined by the grating profile. Also, it was found that both resist (microcavity) thickness and the depth of the fundamental amplitude of the corrugation affected the position in energy of the waveguide modes and the width of the band gaps to a far larger extent than any other parameters. This has enabled accurate values for these two parameters to be obtained, especially for the first two regions where the waveguide modes are comparatively narrow. The parameters used for these plots also showed differences between the values of the fundamental amplitude for the air/silver corrugated interface obtained from the fits of Fig. 3 and those of the photoresist/silver corrugated interface as experienced by the waveguide modes of the structure. This was expected from the widths of the measured band gaps. These values are as follows.

Region 1: $a_{0}=15.0 \pm 0.5 \mathrm{~nm}$, mean photoresist thickness $d_{\mathrm{PR}}=136 \pm 1 \mathrm{~nm}$.

Region 2: $a_{0}=28.0 \pm 0.5 \mathrm{~nm}, \quad d_{\mathrm{PR}}=120 \pm 1 \mathrm{~nm}$.

Region 3: $a_{0}=37.3 \pm 1.0 \mathrm{~nm}, \quad d_{\mathrm{PR}}=104 \pm 1 \mathrm{~nm}$.

Region 4: $a_{0}=39.5 \pm 1.5 \mathrm{~nm}, \quad d_{\mathrm{PR}}=88 \pm 1.5 \mathrm{~nm}$.

Here the region number denotes the least (region 1) to most (region 4) exposure.

All of the previous plots of Fig. 4 examined the situation where light was incident on the sample at an azimuthal angle of $\phi=0^{\circ}$, i.e., the plane of incidence was always perpendicular to the direction of the corrugation grooves and parallel to the Bragg vector of the grating. Previous work has demonstrated experimentally that as the azimuthal angle is increased, the central frequency of the band gap also rises. ${ }^{27}$ It is the $x$ component of the in-plane momentum of the inci- dent light that experiences the periodicity of the surface. For directions other than along the $x$ axis, we denote the total in-plane wave vector by $k_{x z}$. The component of the in-plane wave vector along the $x$ axis can then be given as $k_{x}$ $=k_{x z} \cos (\phi)$. The gap now occurs when $k_{x z}=k_{g} / 2 \cos (\phi)$. Hence, as $\omega \propto k$, we can infer that the angular frequency of the center of the band gap will rise as $1 / \cos (\phi) .{ }^{6}$ Thus, as $\phi$ increases, the position in energy of the lower branch of the band gap overtakes the position of the upper edge at $\phi$ $=0^{\circ}$ : the band gap becomes discontinuous. The value of $\phi$ where this discontinuity occurs depends on the width of the band gap at $\phi=0^{\circ}$; the wider the gap, the higher the value of $\phi$ will be before the gap is discontinued. ${ }^{38}$

With this in mind, the position of the band gap for each of the four regions of the sample was investigated at $\phi=45^{\circ}$. The reason for choosing this azimuthal angle was that a sample with a suitable pair of identical corrugations crossed at $90^{\circ}$ to each other will generate a band gap, which rises to its maximum energy at $\phi=45^{\circ}$ to the Bragg vector of either grating. By providing a suitable depth of corrugation, it should therefore be possible to prevent the band gap from becoming discontinuous at this azimuthal angle. As the position of the band gap will be symmetric about this $\phi=45^{\circ}$ position, a common band gap will then exist for all $\phi$. Figures 7(a) and 7(b) show the measured transmission of region 1 for both TM and TE polarizations, incident at $\phi=45^{\circ}$ to the grating Bragg vector. As can be seen, there is some coupling to SPP modes for both incident polarizations at this azimuthal angle. This is due to the fact that the relative orientation of the surface shape and the electric vector results in the local electric vector having both TE and TM components relative to the surface. However, for the TE case, it can be clearly seen that the strongest coupling is to the upper and lower branches of the waveguide mode band gap, which have remained very close to their $\phi=0^{\circ} \omega$ values [Fig. $4(\mathrm{~b})$ ], indicating continuity of the band gap up to this value of $\phi$. This indicates that even this relatively modest surface 

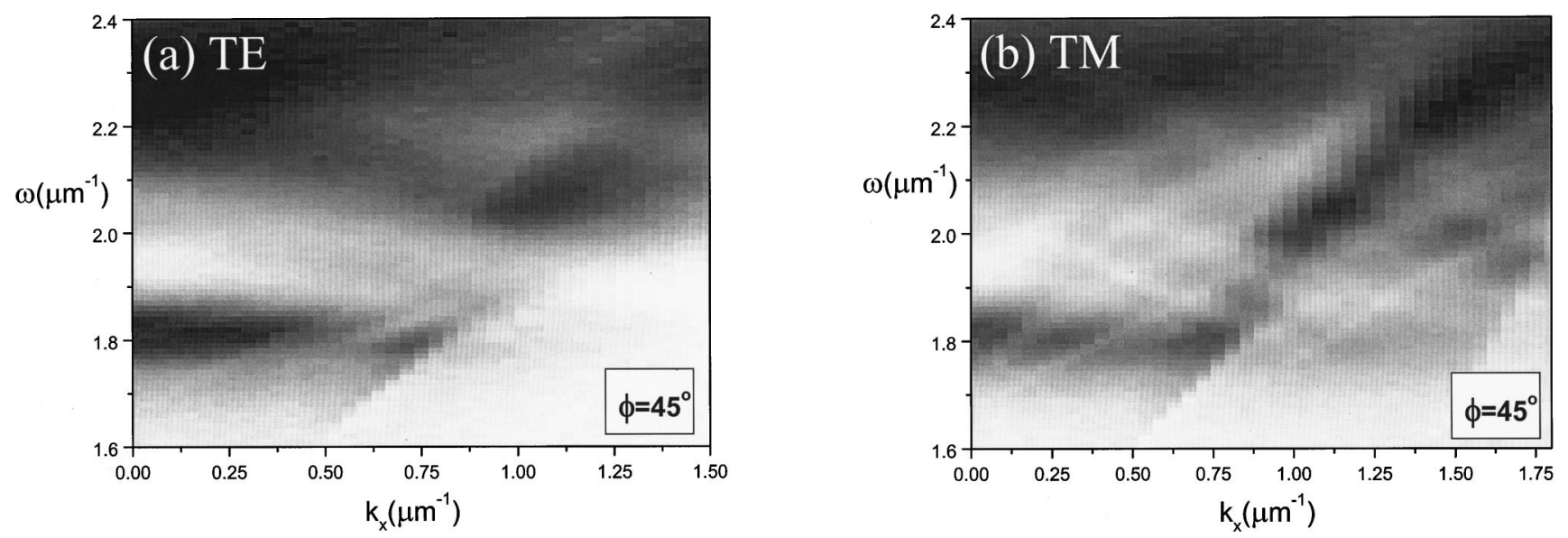

FIG. 8. Transmission gray scales for both polarizations at $\phi=45^{\circ}$ for the doubly corrugated sample. Similar plots were obtained for a range of azimuthal angles. Two-dimensional periodicity prevents the rise in the central frequency of the band gap as $\phi$ is increased. For the TE-polarized mode the band gap is well defined over all azimuths from $\phi=0^{\circ}$ to $90^{\circ}$. Due to surface symmetry, we can thus assume that this remains true for the entire $360^{\circ}$. For TM polarization of (b), an upper and lower branch to the waveguide mode is maintained, at the same $\omega$ values as in the TE-polarized case, but the situation is somewhat complicated by stronger coupling to SPP modes.

modulation should produce a full band gap for TE-polarized waveguide modes if a pair of such corrugations were crossed at $90^{\circ}$ to each other. The TM-polarized transmission of Fig. 7(a) exhibits SPP coupling as seen before, so that distortion to the waveguide mode dispersion can be quite significant. However, an upper and lower branch are still very much evident, positioned at $\omega$ values close to those of the $\phi=0^{\circ}$ case, and so indicates that two-dimensional texturing of a suitable depth should be able to maintain an average position for an upper and lower branch for the TM waveguide mode.

Figures $8(\mathrm{a})$ and $8(\mathrm{~b})$ show the transmitted intensity for both TB and TM polarizations for the second sample, at the azimuthal angle $\phi=45^{\circ}$ to one of the grating Bragg vectors. Very similar plots were obtained for a range of azimuthal angles from $\phi=0^{\circ}-90^{\circ}$. The texture of this sample was produced using the double-exposure technique outlined in Sec. II. The second Bragg vector introduced by the second grating reduces the dimensionality of the system still further and prevents the continued rise in energy of the central frequency of the band gap past $\phi=45^{\circ}$. Away from a plane of incidence that is parallel to either set of grating grooves, both corrugations contribute to the formation of the band gap. Due to the thickness of the resist, the band structure around the band gap is in a region of higher absorption for the photoresist cavity. This leads to broadening of the modes. However, in the TE-polarized case the upper and lower band edges are visible at $\omega^{-} \approx 1.8 \mu \mathrm{m}^{-1}$ and $\omega^{+} \approx 2.1 \mu \mathrm{m}^{-1}$ for $\phi=45^{\circ}$ and barely change from these values throughout the range of azimuthal angles studied, i.e., the band gap can be considered as continuous in $\phi$. As in the case of the first sample, the upper and lower band edges are nearly flat and are accessible at all azimuths, which may have great significance for emission from within such a structure. Strongest coupling to SPP modes, due to the surface shape, is at this azimuthal angle $\left(\phi=45^{\circ}\right)$ but does not heavily influence the band-gap region. The TM-polarized plot exhibits the surface plasmon-polariton coupling and resultant distortion as noted previously, but an average position of an upper and lower branch for the TM waveguide mode can be seen at $\omega^{-}$ $\approx 1.8 \mu \mathrm{m}^{-1}$ and $\omega^{+} \approx 2.05 \mu \mathrm{m}^{-1}$.
Perhaps the most striking aspect of this work is the change of the otherwise continuous dispersion curve of the waveguide modes into a series of discrete and nearly flat bands. For a single corrugation, we have shown that this is the case for surface modulations whose depths constitute approximately $20 \%$ or more of the total waveguide thickness. We can therefore see that there is now a significantly higher density of states for both the TE- and TM-polarized waveguide modes in specific frequency ranges. In other words, a wide range of in-plane wave vector $\left(k_{x}\right)$ values is available for a comparatively narrow range of $\omega$ values for each band. This is significant when one considers the case of an emissive species placed inside such a microcavity structure. For a monochromatic emitter, ${ }^{8}$ emission into waveguide modes is prevented if the emission frequency lies within the band gap of the waveguide mode. Microcavity-enhanced emission from the structure is thus prevented, as the band gap reduces the number of "routes of emission," and it is therefore likely that the lifetime of the emitter will be increased. Conversely, if the emission frequency overlies a significantly flat band edge, we expect a corresponding reduction in the emission lifetime, together with radiation from the structure over a wide range of emission angles. For a broadband emitter ${ }^{25}$ whose emission spectrum may cover a range that overlies a number of these flat bands, we can expect to see enhanced emission into each band and suppression of emission into the band-gap regions. The spectrum of emission from the structure at a particular angle would then be made up of a series of spikes, corresponding to each of the bands that the broadband emission covers, with low emission at the frequencies that cover the band-gap regions. An extension of the singlecorrugation texture to a two-dimensional texture, providing a band gap for all azimuthal angles for at least the TEpolarized waveguide mode, would significantly enhance all the above-mentioned effects still further.

It might be thought that the presence of metallic layers, such as those utilized in the work presented here, is a prohibitive feature of emissive device design due to the relatively high absorption of the metal. This issue is of particular importance when the emitted light must pass through such a 
metal layer in order to escape from the device. However, work carried out in our laboratory demonstrates that in spite of absorption, metallic layers may still provide adequately high- $Q$ cavities with good emissive efficiencies. ${ }^{39}$

It is also important to point out that although the transmission studies that have been presented here give clear information on the nature of the dispersion curve for the waveguide modes, they cannot be used to predict the coupling strength of an emitter to the modes. How well an emitter couples to a particular mode in such systems depends strongly upon the position and orientation of the emitter within the cavity. For example, an emitter placed at the center of a microcavity (i.e., at $d / 2, d$ being the distance between the mirrors) will couple well to the first-order waveguide mode $\left(\omega_{1}\right)$ due to the relatively high in-plane electric field $\left(E_{x}\right)$ of the mode at this position. However, an emitter at $d / 2$ will not couple so well to the second-order waveguide mode $\left(\omega_{2}\right)$, because there is a node for $E_{x}$ at this point for this mode. Hence, the strength of coupling to modes via transmission through such structures does not give a good guide to the strength of coupling via emission from within.

\section{CONCLUSION}

We have demonstrated that periodic texturing of a solidstate, metal-clad $\lambda / 2$ waveguide can generate a band gap in the dispersion of the guided modes. For a single corrugation we investigated how the size of the band gap changes with the depth of modulation and found dramatic modification of the dispersion, leading to the formation of substantially flat bands. The widths of the band gaps generated were sufficient to ensure band-gap continuity in azimuthal angle from $\phi$ $=0^{\circ}-45^{\circ}$. From this we determined a suitable surface profile that provides a complete band gap for all directions of propagation of the TE-polarized waveguide mode. We then investigated the band structure of such a microcavity and have found that a complete band gap for the TE waveguide mode exists and that there is significant alteration to the TM waveguide mode.
The impact such microstructures may have on controlling the emission of light has been discussed in the context of both monochromatic and broadband emissive species. We have suggested that such flat bands will have a significant effect upon the spontaneous emission lifetime of a monochromatic emitter, due to the high mode density associated with such flat bands, and will be most strongly exhibited with the use of a two-dimensional texture. The strong alteration that such cavities may induce in radiation from a typical broadband emitter has also been discussed, notably the enhanced emission at certain frequencies due to the flat bands and suppressed emission over the ranges covered by the band gaps. We have also noted that the flat nature of the band edges should allow devices to be constructed that are insensitive to viewing angle. By using just one corrugation, emission dominated by, say, the band edge of the TE mode should be well polarized. Conversely, where polarized output is not desired, the two-dimensional microstructure examined here may allow the advantages of microstructure, e.g., insensitivity to viewing angle, to be obtained without an associated polarized nature of the source.

We have also noted that the strength of coupling of an emissive species to the modes of such systems is highly dependent on the position and orientation of the emissive species within the microcavity. We are actively pursuing the fabrication of both monochromatic and broadband emitters placed in such one- and two-dimensionally textured microcavity environments in order to study the interaction between emitters and the optical modes of such systems.

\section{ACKNOWLEDGMENTS}

The authors would like to express their thanks to Weichao Tan for his advice on the theoretical modeling. M. G. Salt would also like to thank DERA (Malvern) financial support from CASE. The financial assistance of the EPSRC is also gratefully acknowledged. W. L. Barnes was given financial support by the Leverhulme Trust.
*Present address: Applied Optics Group, Institute of Microtechnology, Rue A.-L. Breguet 2, University of Neuch, Neuch, Switzerland. Electronic address: m.g.salt@ex.ac.uk

${ }^{1}$ Y. Yamamoto and R. E. Slusher, Phys. Today 46 (6), 66 (1993).

${ }^{2}$ I. Abram, I. Robert, and R. Kuszelewicz, IEEE J. Quantum Electron. 34, 71 (1998).

${ }^{3}$ F. B. Seeley, J. E. Alexander, R. W. Connatser, J. S. Conway, and J. P. Dowling, Am. J. Phys. 61, 545 (1993).

${ }^{4}$ A. Dodabalapur, L. J. Rothberg, R. H. Jordan, T. M. Miller, R. E. Slusher, and J. M. Phillips, J. Appl. Phys. 80, 6954 (1996).

${ }^{5}$ H. Rigneault, C. Amra, C. Begon, M. Cathelinaud, and C. Picard, Appl. Opt. 38, 3602 (1999).

${ }^{6}$ S. C. Kitson, W. L. Barnes, and J. R. Sambles, J. Appl. Phys. 84, 2399 (1998).

${ }^{7}$ I. P. Kaminow, W. L. Mammel, and H. P. Weber, Appl. Opt. 13, 396 (1974).

${ }^{8}$ P. T. Worthing, R. M. Amos, and W. L. Barnes, Phys. Rev. A 59, 865 (1999).

${ }^{9}$ P. T. Worthing and W. L. Barnes, J. Opt. Soc. Am. A 1, 501 (1999).
${ }^{10}$ R. E. Slusher, Solid State Commun. 92, 149 (1994).

${ }^{11}$ I. Abram and G. Bourdon, Phys. Rev. A 54, 3476 (1996).

${ }^{12}$ E. Yablonovitch, J. Opt. Soc. Am. B 10, 283 (1993).

${ }^{13}$ A. van Blaaderen, R. Ruel, and P. Wiltzius, Nature (London) 385, 321 (1997).

${ }^{14}$ K. E. Paul, T. L. Breen, J. Aizenberg, and G. M. Whitesides, Appl. Phys. Lett. 73, 2893 (1998).

${ }^{15}$ G. Gigli, R. Rinaldi, C. Turco, P. Visconti, and R. Cingolani, Appl. Phys. Lett. 73, 3926 (1998).

${ }^{16}$ H. Schmid, H. Biebuyck, B. Michel, and O. J. F. Martin, Appl. Phys. Lett. 72, 2379 (1998).

${ }^{17}$ S. C. Kitson, W. L. Barnes, J. R. Sambles, IEEE Photonics Technol. Lett. 8, 1662 (1996).

${ }^{18}$ R. K. Lee, O. J. Painter, B. D’Urso, A. Scherer, and A. Yariv, Appl. Phys. Lett. 74, 1522 (1999).

${ }^{19}$ S. C. Kitson, W. L. Barnes, and J. R. Sambles, Phys. Rev. Lett. 77, 2670 (1996)

${ }^{20}$ H. Rigneault, F. Lemarchand, A. Sentenac, and H. Giovannini, Opt. Lett. 24, 148 (1999).

${ }^{21}$ R. Windisch, P. Heremans, A. Knobloch, P. Kiesel, G. H. Döhler, 
B. Dutta, and G. Borghs, Appl. Phys. Lett. 74, 2256 (1999).

${ }^{22}$ S. Fan, P. R. Villeneuve, and J. D. Joannopoulos, Phys. Rev. Lett. 78, 3294 (1997).

${ }^{23}$ M. Meier, A. Mekis, A. Dodabalapur, A. Timko, R. E. Slusher, J. D. Joannopoulos, and O. Nalamasu, Appl. Phys. Lett. 74, 7 (1999).

${ }^{24}$ R. Zengerle, J. Mod. Opt. 34, 1589 (1987).

${ }^{25}$ H. Benisty, H. De Neve, and C. Weisbuch, IEEE J. Quantum Electron. 34, 1632 (1998).

${ }^{26}$ E. Yablonovitch, Phys. Rev. Lett. 58, 2059 (1987).

${ }^{27}$ M. G. Salt, and W. L. Barnes, Opt. Commun. 166, 151 (1999).

${ }^{28}$ A. Dodabalapur, Solid State Commun. 102, 259 (1997).

${ }^{29}$ M. C. Hutley, Diffraction Gratings (Academic, London, 1982).

${ }^{30}$ R. A. Watts, J. B. Harris, A. P. Hibbins, T. W. Preist, and J. R. Sambles, J. Mod. Opt. 43, 1351 (1996).

${ }^{31}$ R. M. A. Azzam and N. M. Bashara, Ellipsometry and Polarized
Light (North-Holland, Amsterdam, 1977).

${ }^{32}$ E. L. Wood, J. R. Sambles, N. P. Cotter, and S. C. Kitson, J. Mod. Opt. 42, 1343 (1995).

${ }^{33}$ R. A. Watts, J. R. Sambles, and M. C. Hutley, J. Mod. Opt. 45, 639 (1998).

${ }^{34}$ N. P. K. Cotter, T. W. Preist, and J. R. Sambles, J. Opt. Soc. Am. A 12, 1097 (1995).

${ }^{35}$ S. J. Elston, G. P. Bryan-Brown, and J. R. Sambles, Phys. Rev. B 44, 6393 (1991).

${ }^{36}$ J. Chandezon, M. T. Dupuis, G. Cornet, and D. Maystre, J. Opt. Soc. Am. 72, 839 (1982).

${ }^{37}$ W. L. Barnes, J. Mod. Opt. 45, 661 (1998).

${ }^{38}$ W. L. Barnes, S. C. Kitson, T. W. Preist, and J. R. Sambles, J. Opt. Soc. Am. 14, 1654 (1997).

${ }^{39}$ P. T. Worthing, J. A. E. Wasey, and W. L. Barnes (unpublished). 